\title{
Design of A Regional perimeter Optical fiber Security and Protection System
}

\author{
Guofan Chen and ShuangXin $\mathrm{Wu}$ \\ School of Mechanical Engineering, Anhui University of Science and Technology, Huainan, China
}

\begin{abstract}
The current commonly used widespread in electronic Security and Protection System Widespread the high cost of maintenance,Security distance short,Susceptible to electromagnetic interference.Aim at these problems, this paper based on source of access to separate The invasion of positioning signal and The invasion characteristic signal ,Design of a Regional perimeter Optical fiber Security and Protection System.The system can significantly improve the positioning accuracy, reduce the rate of false positives.
\end{abstract}

\section{Introduction}

At present, domestic Electronic security system include optical fiber probe, infrared detection, geomagnetism detection and closed-circuit television, and the latter three ones due to the limitation of the power supply, they are usually security distance is short, are susceptible to electromagnetic interference, high maintenance cost disadvantage [1]. Because the distributed optical fiber security technology can overcome the above shortcomings and has had a rapid development in recent years, however, there are still some shortcomings in the current distributed optical fiber security technology [2]. Positioning accuracy is mostly outside the $20 \mathrm{~m}$, not suitable for the military and sensitive department of security requirements; to wind sways grass, animal intrusion events Characteristic can't accurate analysis, lead to high rate of false positives [3]. So it is necessary to research on optical fiber security invasion of precise positioning ( 0.5 meters) technology, reduce the intrusion event False alarm rate and research and development of its auxiliary equipment.

This system use the TDC (Time digital conversion technology) to realize the super short time difference (picosecond level) measurements, and solved the traditional measuring method in a short period of time is difficult to apply problem, and heightens the precision of Intrusion place location. When measuring time interval TDC measure the pulse signal converted into digital signals, don't need to do A/D conversion, Single-chip microcomputer connected to converter could have completed single chip microcomputer control of the converter and to deal with the results of the measurement [4]. TDC resolution can reach $65 \mathrm{ps}$, namely every measurement error is less than $0.98 \mathrm{~cm}$.

\section{System structure}

The AT89C52 single chip microcomputer is the control core in this system,Mainly includes: the laser generator,optical coupler,four fiber cable,the photoelectric converter, electrical signal processing module,TDC, SCM and PC used as a upper computer,as shown in Figure 1.

\subsection{System works}

The signal source of the system come from the optical signals generated by the laser generator, After the laser beams was subjected to a series of Optical coupler action,then the photoelectric converter converts laser interference signals into electrical signals, the system uses four core optical fiber as the sensing device accept outside disturbance, The intrusion events generate signals on the optical cable, and input to the system for signal processing.then Electrical signal processing module output pulse signal. This time difference is converted to digital information by TDC,The disturbance signal emitted in both directions in a closed loop four core cable system, Thereby generating the pulse signal of a time difference $\mathrm{T}$,

Through single chip microcomputer program for processing,And then the results transmitted to the upper computer display. 


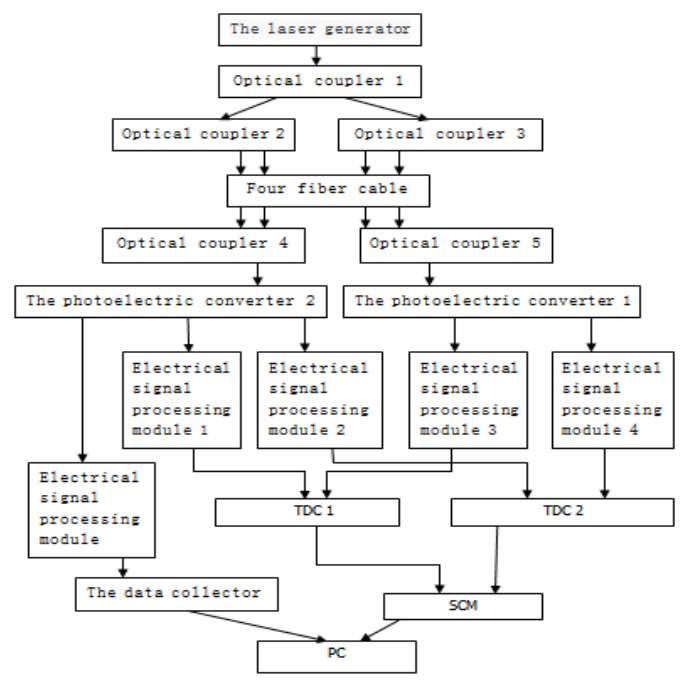

Fig. 1. System structure diagram.

\subsection{System implementation process targeting}

When the invasion of disturbance signal is stable, it is converted into stable electrical signals. The upper computer neither displays the invasion position nor gives an alarm. When the invasion occur, saltation light signals will be generated on intrusion location, and the volatility information would be obtained go through a series of signal processing process, and then the photoelectric conversion system and signal processing module can output the trigger pulse signal, lead to trigger the TDC work. Eventually,the computer calculates the location of the specific locations intrusion incident, and then Invasion of positioning is completed.

\section{System program design}

As soon as the system just starts, its initializes the interface of system and set parameters of a variety of hardware interfaces. Then enter the main loop program, the data can be analyzed, transmitted, analyzed and processed. continuous monitoring of the signature of four-core optical fiber, it continuous monitoring of the signature of four-core optical fiber, If there is an intrusion event occur, The system will respond immediately. The results are finally displayed on the upper computer. Accomplish Positioning function of the system.

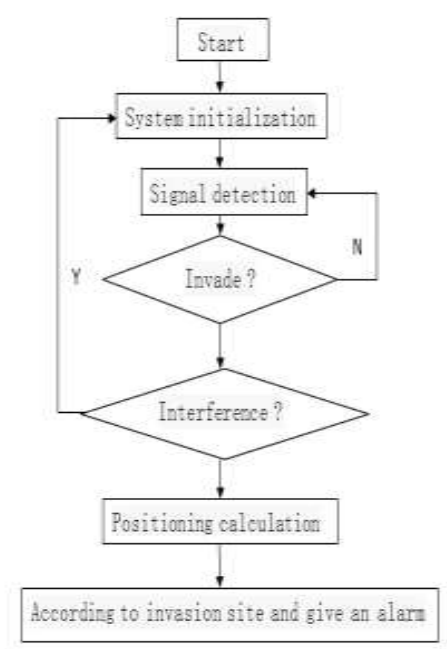

Fig. 2. Software program flowchart. 


\section{Conclusion}

This region fiber perimeter security system adopts kernel 51 single-chip microcomputer as a core of Control Processor. the system uses the four-core cable device to obtain signal feature of intrusion incident. Invasion signal transforms the optical signals into electrical signals by the Optical coupler and Signal processing module. That pass through the electrical signal processing module and completed the signal processing by the TDC. Data acquisition card acquire system data and then transmits that to a upper computer. Finally the computer finish identify intrusion event properties.

The technical characteristics of this system includes innovation based on TDC invasion of the positioning system ,based on intrusion short time Fourier transform and wavelet analysis for Signal Feature Extraction and Intrusion alarm based on database technology.

\section{References}

1. Guangming Luo, Chunxi Zhang, etc. Distributed sensing and positioning technology fiber optic security system Piezoelectrics and Acoustooptics, 2009,31 (3): 327-333

2. Suiren Wan, Licheng Peng research extraction and classification algorithm security system features fiber optic signal Science \& Technology Review, 2012,30 (36): 24-28

3. xing-qun zhao, Dongqing Xue, Distributed optical fiber vibration sensor signal data acquisition system Science and Technology News,2012,30(18):45-49

4. GuoFan Chen, Dawen Wang, Xiaoyan Wang, A regional fiber optic perimeter security systems. China Patent, 201220161011.2012-10-31. 